\title{
Dysregulated long noncoding RNAs in the brainstem of the DBA/1 mouse model of SUDEP
}

\author{
Deng Chen, Lina Zhu, Xin Lin, Dong Zhou* and Ling Liu*
}

\begin{abstract}
Background: Long noncoding RNAs (IncRNAs) play an important role in many neurological diseases. This study aimed to investigate differentially expressed IncRNAs and messenger RNAs (mRNAs) in the susceptibility gaining process of primed DBA/1 mice, a sudden unexpected death in epilepsy (SUDEP) model, to illustrate the potential role of IncRNAs in SUDEP.
\end{abstract}

Methods: The Arraystar mouse IncRNA Microarray V3.0 (Arraystar, Rockville, MD) was applied to identify the aberrantly expressed IncRNAs and mRNAs between primed DBA/1 mice and normal controls. The differences were verified by qRT-PCR. We conducted gene ontology (GO), the Kyoto Encyclopedia of Genes and Genomes (KEGG) pathway and coexpression analyses to explore the possible function of the dysregulated RNAs.

Results: A total of 502 IncRNAs (126 upregulated and 376 downregulated IncRNAs) and 263 mRNAs (141 upregulated and 122 downregulated mRNAs) were dysregulated with $P<0.05$ and a fold change over 1.5, among which Adora3 and Gstt4 were possibly related to SUDEP. GO analysis revealed that chaperone cofactor-dependent protein refolding and misfolded protein binding were among the top ten downregulated terms, which pointed to Hspa1a, Hspa2a and their related IncRNAs. KEGG analysis identified 28 upregulated and 10 downregulated pathways. Coexpression analysis showed fifteen dysregulated long intergenic noncoding RNAs (lincRNAs) and three aberrantly expressed antisense IncRNAs, of which AK012034 and NR_040757 are potentially related to SUDEP by regulating $L M N B 2$ and ITPR1, respectively.

Conclusions: LncRNAs and their coexpression mRNAs are dysregulated in the priming process of DBA/1 in the brainstem. Some of these mRNAs and IncRNAs may be related to SUDEP, including Adora3, Lmnb2, Hspa1a, Hspa1b, Itrp1, Gstt4 and their related IncRNAs. Further study on the mechanism of IncRNAs in SUDEP is needed.

Keywords: Long noncoding RNAs, SUDEP, Epilepsy, DBA/1 mouse

\section{Background}

Long noncoding RNAs (lncRNAs) were initially considered a byproduct of RNA polymerase II and as the "noise" of gene transcription without biological function [1]. However, recent studies have described that a large

\footnotetext{
*Correspondence: zhoudong66@yahoo.de; neurologyliuling@163.com; zjllxx1968@163.com

Department of Neurology, West China Hospital, Sichuan University, Wai Nan Guo Xue Lane 37 \#, 610041 Chengdu, Sichuan, China
}

number of lncRNAs have critical roles in chromatin modification and transcriptional and epigenetic regulation $[1,2]$. LncRNAs are associated with many physiological processes in the central nervous system, including neurogenesis, regulation of neurotransmitters, ion channels and synaptic plasticity [3]. Specifically, accumulating evidence has demonstrated that lncRNAs are involved in epilepsy. In 2015, Lee et al. confirmed that the expression of lncRNAs is different between epilepsy

(c) The Author(s). 2021 Open Access This article is licensed under a Creative Commons Attribution 4.0 International License, which permits use, sharing, adaptation, distribution and reproduction in any medium or format, as long as you give appropriate credit to the original author(s) and the source, provide a link to the Creative Commons licence, and indicate if changes were made. The images or other third party material in this article are included in the article's Creative Commons licence, unless indicated otherwise in a credit line to the material. If material is not included in the article's Creative Commons licence and your intended use is not permitted by statutory regulation or exceeds the permitted use, you will need to obtain permission directly from the copyright holder. To view a copy of this licence, visit http://creativecommons.org/licenses/by/4.0/ The Creative Commons Public Domain Dedication waiver (http://creativecommons.org/publicdomain/zero/1.0/) applies to the data made available in this article, unless otherwise stated in a credit line to the data. 
models induced by kainic acid or pilocarpine and normal controls [4]. More recent findings have also indicated that abnormally methylated lncRNAs and their related downstream pathways are involved in the development and progression of temporal lobe epilepsy [5]. Furthermore, Hsiao reported that targeting the lncRNA SCN1ANAT can improve the seizure phenotype in one animal model of Dravet syndrome [6]. These findings demonstrated that IncRNA studies could provide insight into the mechanism and treatment of epilepsy.

Sudden unexpected death in epilepsy (SUDEP) is one of the most common causes of death in epilepsy [7]. However, its underlying mechanism is largely unknown. The occurrence of SUDEP is highly associated with the brainstem. Patodia et al. reported that in SUDEP postmortem cases, pathological changes have been observed in brainstem respiratory nuclei [8]. Recent MRI studies also identified brainstem volume loss in SUDEP cases [9, 10]. Regarding the molecular mechanism, clinical and laboratory evidence have also pointed out that dysfunction of the serotonin system, adenosine receptors and ion channels in the brainstem are related to SUDEP [11-14]. These findings suggest a brainstem-centered structural basis for SUDEP. More importantly, similar pathological findings have been observed in non-SUDEP death cases with epilepsy [8]. The brainstem of accidental nonSUDEP death in epilepsy cases showed intermediate status between healthy controls and SUDEP, suggesting that repeat seizures could somehow remodel the brainstem $[8,15,16]$ and, from a pathological aspect, that structural 'susceptibility' to SUDEP may accumulate through this process. Thus, we suspected that epigenetic regulation could participate in this process.

The DBA/1 mouse is a reliable animal model for SUDEP [11]. It is characterized by audiogenic seizures (AGSz), followed by seizure-induced respiratory arrest (S-IRA) and death [17]. Similar sequential events of death immediately following a terminal seizure, which led to respiratory arrest then death, have been observed in SUDEP and near-SUDEP cases [8, 18-22]. Moreover, a variety of studies regarding the DBA/1 mouse SUDEP model have illustrated that several brainstem regions (including periaqueductal gray matter, the respiratory complex and raphe nuclei) are associated with SUDEP [23-25]. Interestingly, the susceptibility to S-IRA of DBA/1 increases when given sound stimulation on PND 21 to 30 (which is called priming). Once primed, such susceptibility will last until PND 100 [26]. This distinct feature of DBA/1 makes it unique compared to other SUDEP models, and epigenetic regulation in this process is also speculated. Thus, studying the priming process on DBA/1 could provide insight into the SUDEP mechanism from an epigenetic perspective.
In light of these findings, we hypothesized that IncRNAs participate in the process of gaining susceptibility of S-IRA in DBA/1 and SUDEP. We investigated the expression profiles of lncRNAs and messenger RNAs (mRNAs) in the brainstem of primed and normal DBA/ 1 by microarray analysis. Assessing the potential role of IncRNAs in increasing susceptibility to S-IRA may provide new insight into the potential mechanism and therapeutic targets of SUDEP.

\section{Methods \\ Animals}

The animal experiments were performed in accordance with the Guidelines of Animal Care and Use Committee of Sichuan University. Male, postnatal day (PND) 21-23 DBA1 mice were obtained from the Charles River Laboratories Experimental Animal Center (Beijing, China) and housed in specific pathogen free (SPF) conditions for 1 week prior to experimentation. The DBA/1 mice had access to food and water and were kept under standard lighting conditions (12-h light/dark cycle).

\section{Seizure priming and seizure-induced sudden death}

DBA/1 mice at 28 PND (10-15 g) were used in the experiments. An electric bell (120 dB SPL) (SCF 8, MinRong's Electronics, China) was used to induce generalized audiogenic seizures (AGSz) in the experimental group. Each mouse was placed in a $40-\mathrm{cm}$ diameter cylindrical plastic container and was given acoustic stimulus lasting for $60 \mathrm{~s}$ or until the onset of a seizure. Some mice could develop seizure-induced respiratory arrest (S-IRA) during seizures. The rodent respirator was used to rescue the mice with S-IRA by resuscitating within $5 \mathrm{~s}$ after the final respiratory gasp, as reported previously [26]. We stimulated each mouse three times a day for a week. If any mice presented with S-IRA, the rest of the stimulation on that day was cancelled, and the mice rested for $24 \mathrm{~h}$.

\section{Grouping}

The mice that experienced S-IRAs and were successfully rescued more than three times during priming were considered a SUDEP model. Normal controls were those without acoustic stimulation. At PND 36 (24 h after the last stimulation, all had seizures the last day), the SUDEP model and normal mice (four for each group) were decapitated under anesthesia with isoflurane inhalation, and the brains were removed immediately. We used a sharp surgical blade to separate the whole brainstem between bregma $-3 \mathrm{~mm}$ and bregma $-9 \mathrm{~mm}$ based on a previous report [23]. 


\section{Total RNA extraction}

The brainstems were immediately frozen in liquid nitrogen and transferred to a $-80{ }^{\circ} \mathrm{C}$ refrigerator for later use. According to the manufacturer's instructions, total RNA was extracted using TRIzol reagent (Invitrogen Life Technologies, Carlsbad, CA). A NanoDrop ND-1000 spectrometer (Thermo Fisher Scientific, USA) was used to determine the quantity and quality of extracted RNA. The standard denaturing agarose gel electrophoresis method was applied to measure RNA integrity.

\section{Microarray analysis}

We used Arraystar mouse lncRNA Microarray V3.0 (Arraystar, Rockville, MD), containing 35,923 lncRNAs and 24,881 coding transcripts, to describe the lncRNAs and protein coding transcripts of the mice. Sample labeling and array hybridization were performed following the Agilent One-Color Microarray-Based Gene Expression Analysis protocol (Agilent Technology) with slight changes. After removing rRNA from total RNA (RNAONLY Eukaryotic RNA Isolation Kit, Epicenter), purified mRNA was obtained. We amplified each sample into fluorescent cRNA by using random primers (Arraystar Flash RNA Labeling Kit, Arraystar). Then, we used an RNeasy Mini Kit (Qiagen) to purify labeled cRNAs and a NanoDrop ND-1000 to detect the concentration and activity. The labeled cRNA was hybridized onto microarray slides and then washed, fixed and scanned successively.

\section{Quantitative real-time PCR}

Four extra SUDEP models and 4 controls were prepared following the same protocol in "Animals" "Seizure priming and seizure-induced sudden death" "Grouping" for this test. We used TRIzol reagent (Invitrogen Life Technologies) to extract the total RNA from frozen brainstem tissues and SuperScript ${ }^{\text {TM }}$ III Reverse Transcriptase (Invitrogen) to transcribe cDNA following the manufacturer's protocol. Dysregulated lncRNAs were examined by quantitative real-time PCR (qRT-PCR) using the SYBR green PCR kit and the ViiA 7 Real-time PCR system (Applied Biosystems). GAPDH served as an internal control for normalization. A sample of each mouse was tested in triplicate. For each target lncRNA, we first used a tenfold serial dilution (from 1 to $10^{6}$ ) to establish a standard curve to optimize the reaction conditions. Amplification primers are shown in Table 1. The differentially expressed lncRNAs in the SUDEP model group were measured by fold change relative to the control group, calculated as follows:

$$
\text { Fold change }=2^{-(\Delta \Delta \mathrm{Ct} \text { SUDEP }-\Delta \Delta \mathrm{Ct} \text { Control })}
$$

\section{Data analysis}

We used an Agilent DNA Microarray scanner (part number G2505C) to scan the hybridized images and Agilent Feature Extraction software (version 11.0.1.1) to analyze the acquired array images. The GeneSpring GX v12.1 software package (Agilent Technologies) was applied to perform quantile normalization and further data processing. After quantile normalization, all intensities underwent $\log 2$ transformation for further statistical analysis. The $\mathrm{P}$ value was calculated by unpaired $\mathrm{t}$-test based on these normalized and transformed intensities. $P<0.05$ and fold change $\geq 1.5$ were used to identify statistically significant dysregulated lncRNAs and mRNAs. The results were presented by scatter plots. Clustering analysis was performed to show the different expression patterns of lncRNAs and mRNAs in different groups. Gene ontology (GO) analysis was applied to investigate their molecular functions, biological processes and cellular components. Pathway analysis of differentially expressed mRNAs was explored using the Kyoto Encyclopedia of Genes and Genomes (KEGG) database. Coexpression analysis was created to identify differentially expressed antisense lncRNAs with their related sense mRNAs and long intergenic noncoding RNAs (lincRNAs) with their nearby $(<300 \mathrm{~kb})$ coding genes.

Table 1 Primers for qRTPCR

\begin{tabular}{|c|c|c|}
\hline Seqname & Primers $\left(5^{\prime} 3^{\prime}\right)$ & Amplicon size (bp) \\
\hline uc007urg.1 & $\begin{array}{l}\text { F: TAGCAGTGGTGCCTGTGAC } \\
\text { R: GATGGACTCAGGAGGGTCAT }\end{array}$ & 131 \\
\hline AK029922 & $\begin{array}{l}\text { F: GTCTTCTCCCGTTGGCTTCTATC } \\
\text { R: CTATCTGGGCTTATCTTGAGCAGAT }\end{array}$ & 179 \\
\hline ENSMUST00000152600 & $\begin{array}{l}\text { F: TAAGCCCTAGATGGATGTGT } \\
\text { R: TACCAGTATGGGTCCCTAAA }\end{array}$ & 132 \\
\hline ENSMUST00000172531 & $\begin{array}{l}\text { F: GCCGTGTTTCACCCTTCTT } \\
\text { R: CACGCCACTGCCGATITT }\end{array}$ & 281 \\
\hline GAPDH & $\begin{array}{l}\text { F: CACTGAGCAAGAGAGGCCCTAT } \\
\text { R: GCAGCGAACTTTATTGATGGTATT }\end{array}$ & 144 \\
\hline
\end{tabular}




\section{Results}

Overview of differentially expressed IncRNAs and mRNAs There were 502 lncRNAs and 263 mRNAs found to be dysregulated. A total of 126 lncRNAs were upregulated and 376 lncRNAs were downregulated, while 141 mRNAs were upregulated and 122 were downregulated. A heat map and hierarchical clustering analysis of lncRNAs and mRNAs between normal DBA/1 mice and the SUDEP group are shown in Fig. 1. Scatter plots showing the variation in lncRNA and mRNA expression between the two groups are also depicted in Fig. 2. The top 10 dysregulated lncRNAs and mRNAs are summarized in Tables 2 and 3. Specifically, no mRNA related to serotonin or ion channels (potassium, sodium, and calcium) was found to be different. However, adenosine receptor 3 was downregulated in the SUDEP group (Gene symbol: $A D O R A 3$, fold change 2.03 down, $P=$ 0.043). Other RNAs of special interest included Hspala (fold change 9.64 down, $P<0.001$ ), Hspa1b (fold change 3.20 down, $P<0.001$ ), Itrp1 (fold change 1.54 up, $P=$ 0.039 ), and Lmnb2 (fold change 1.78 down, $P=0.002$ ) on the mRNA; NR_040757 (fold change 1.56 down, $P=$ 0.037), Gstt4 (fold change 1.51 down, $P=0.027$ ), AK012034 (fold change 1.86 down, $P=0.025$ ), and ENSMUST00000172531 (fold change 2.60 down, $P=$ $0.010)$ were on lncRNA.

\section{Gene Ontology (GO) analysis}

To elucidate the function of differentially expressed mRNAs, GO analysis was applied to assess the biological processes, cellular components, and molecular functions. The most enriched GO terms corresponding to the upregulated genes were antigen processing and presentation of peptide antigen via the MHC class (GO:0002474, $P<0.001$, Fig. 3A) in biological processes, the MHC class I protein complex (GO:0042612, $P<0.001$, Fig. 3C) in cellular components and beta-2-microglobulin binding (GO:0030881, $P<0.001$, Fig. 3E) in molecular functions. The top enriched GO terms according to downregulated genes were regulation of hormone levels (GO:0010817, $P<0.001$, Fig. 3B) in biological processes, intracellular (GO:0005622, $P=0.001$, Fig. 3D) in cellular components
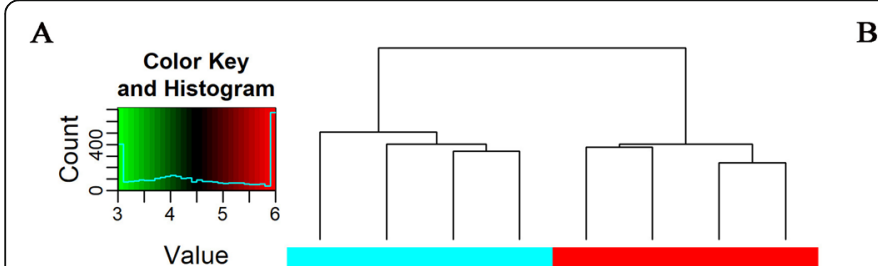

B
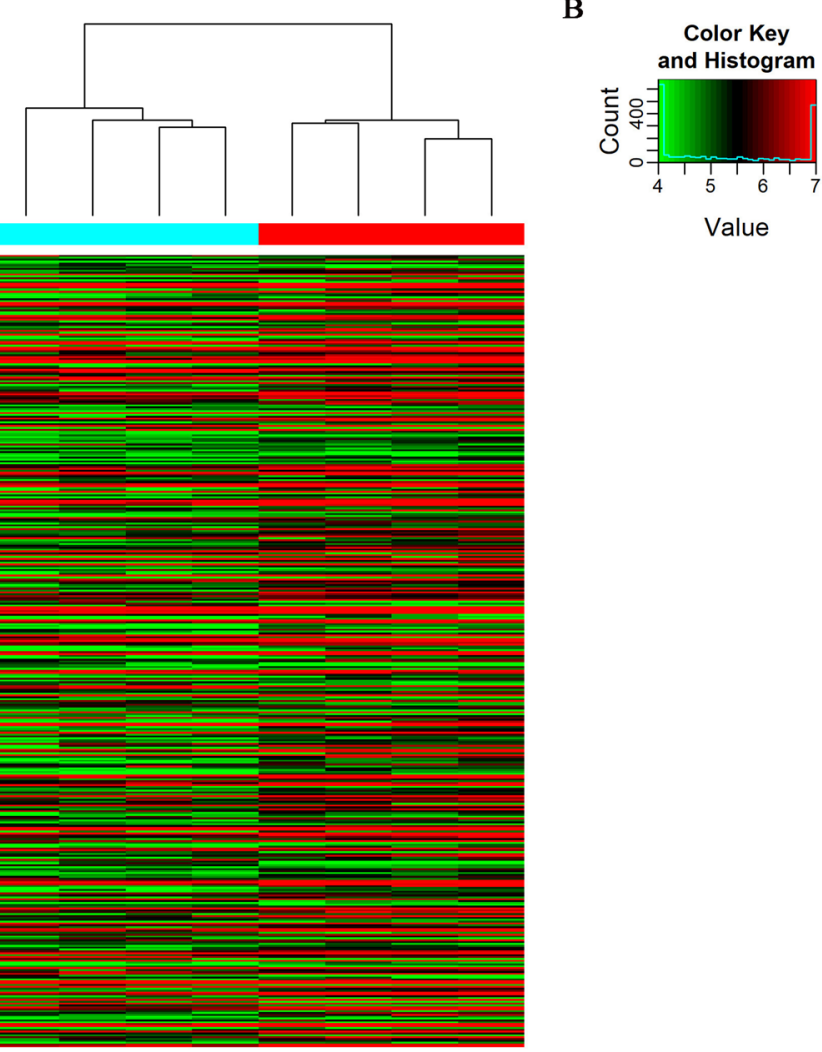

Value

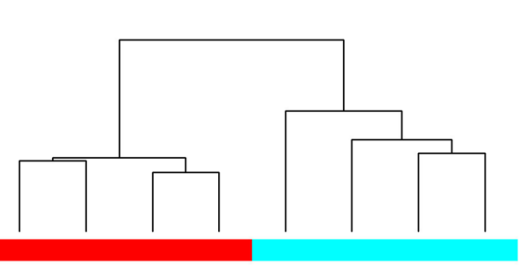

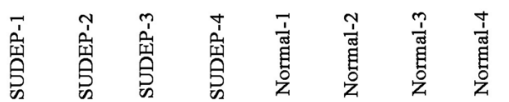

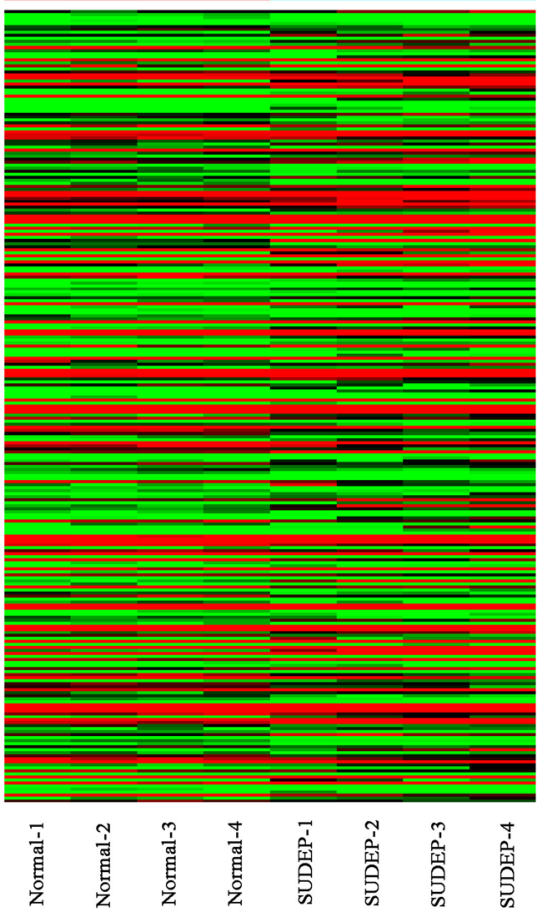

Fig. 1 A Heat map and hierarchical clustering of IncRNA profile comparison between SUDEP group and normal group; B Heat map and hierarchical clustering of mRNA profile comparison between SUDEP group and normal group. Red color indicates high expression and green color indicates low expression 

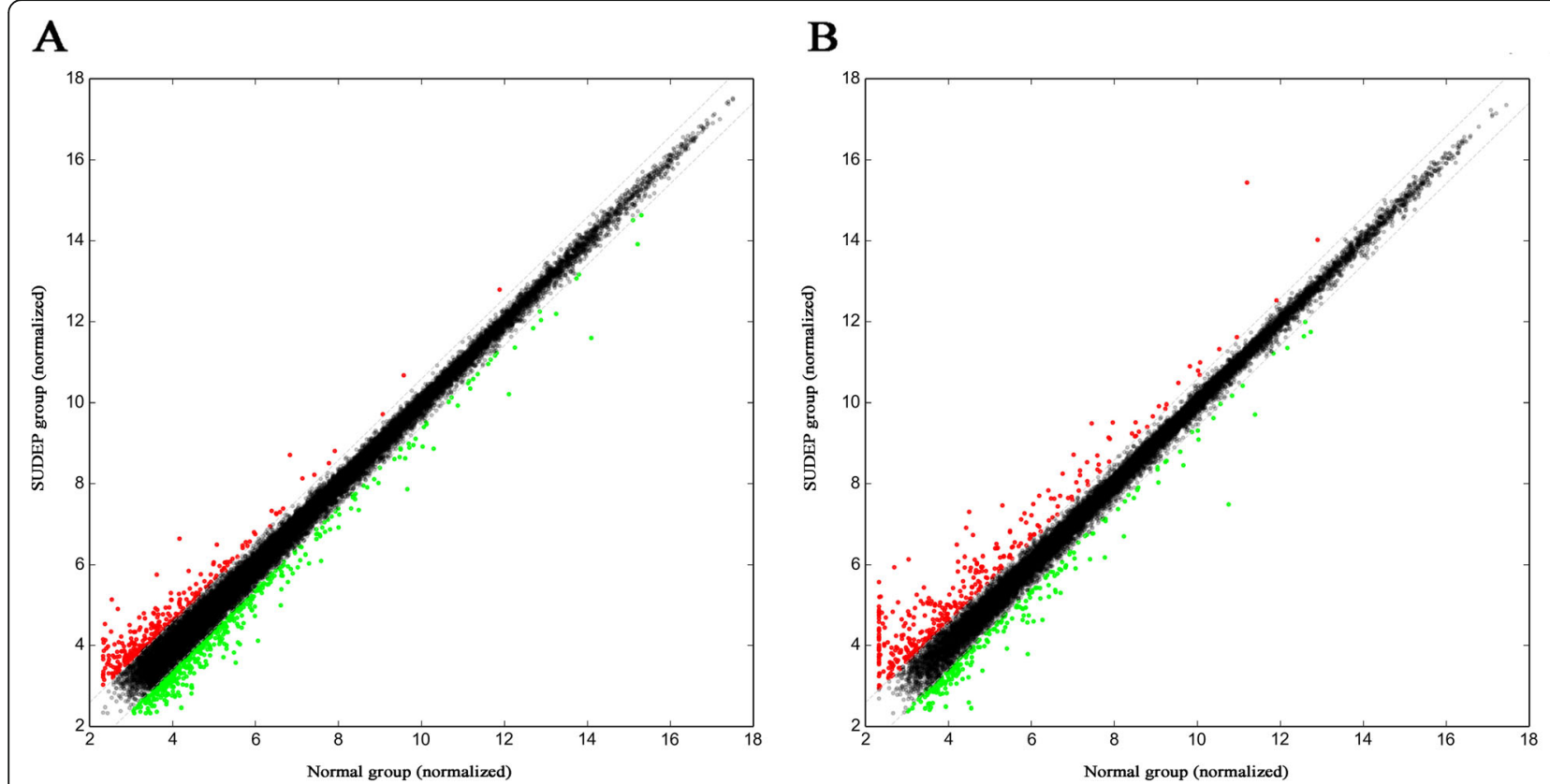

Fig. 2 The scatter plots of IncRNA (A) and mRNA (B) expression between SUDEP group and normal group. The values of the $X$ and $Y$ axes are normalized in the two groups (log2-scaled). The red color and green color above the top grey line or below the bottom grey line represent upor down-regulated genes respectively, which change more than 1.5 fold $(P<0.05)$ between the two groups

and misfolded protein binding (GO:0051787, $P<0.001$, Fig. 3F) in molecular functions. This part of the results is presented in Fig. 3.

\section{Pathway analysis}

Pathway analysis was performed to obtain the biological pathways of the differentially expressed mRNAs based on the Kyoto Encyclopedia of Genes and Genomes (KEGG) database. In contrast with the normal group, we identified 28 upregulated and 10 downregulated pathways in the SUDEP group. For the upregulated genes, the most highly enriched biological process was allograft rejection, while the Fanconi anemia pathway was the top downregulated pathway. The top 10 dysregulated pathways are shown in Fig. 4.

\section{Coexpression analysis}

Three differentially expressed natural antisense lncRNAs (AK012034, AK032393, and uc007ovn.2) and their paired coding genes (Lmnb2, Zmym1, and Ifi27) were identified. The details are described in the supplementary material (see Additional file 1: Table 1).

Table 2 Top 10 differentially expressed IncRNAs in SUDEP group compared with normal group

\begin{tabular}{|c|c|c|c|c|c|c|c|}
\hline \multicolumn{4}{|l|}{ Up-regulated IncRNAs } & \multicolumn{4}{|c|}{ Down-regulated IncRNAs } \\
\hline Seqname & Gene symbol & $\begin{array}{l}\text { Fold } \\
\text { Change }^{a}\end{array}$ & $\overline{\text { Chromosome }}$ & Seqname & Gene symbol & $\begin{array}{l}\text { Fold } \\
\text { Change }^{a}\end{array}$ & $\overline{\text { Chromosome }}$ \\
\hline uc007urg.1 & AK013627 & 5.5312972 & 14 & uc.352- & uc.352 & 3.8636426 & 14 \\
\hline ENSMUST00000118822 & Rpl7|1-ps1 & 4.3902715 & $x$ & AK029922 & AK029922 & 3.3637177 & 6 \\
\hline ENSMUST00000161007 & Gm15717 & 3.6814342 & 8 & humanlincRNA1929- & humanlincRNA1929 & 3.1524491 & 10 \\
\hline AK089422 & AK089422 & 3.5432344 & 15 & ENSMUST00000141932 & Lrrc57 & 3.0635987 & 2 \\
\hline humanlincRNA1945- & humanlincRNA1945 & 3.3141253 & 10 & AK080458 & AK080458 & 3.0231669 & 13 \\
\hline AK050947 & AK050947 & 2.8759742 & 12 & ENSMUST00000117916 & Gm9850 & 2.8692668 & 5 \\
\hline mouselincRNA0735+ & mouselincRNA0735 & 2.8726083 & 17 & ENSMUST00000139399 & Rara & 2.8357532 & 11 \\
\hline AK042193 & AK042193 & 2.8206605 & 1 & AK035001 & AK035001 & 2.7316083 & 10 \\
\hline AK013439 & AK013439 & 2.746032 & 2 & ENSMUST00000172531 & 1110038B12Rik & 2.6065826 & 17 \\
\hline ENSMUST00000152011 & Car12 & 2.5985299 & 9 & uc007ysi.1 & AK020351 & 2.5099841 & 16 \\
\hline
\end{tabular}


Table 3 Top 10 differentially expressed mRNAs in SUDEP group compared with normal group

\begin{tabular}{|c|c|c|c|c|c|c|c|}
\hline \multicolumn{4}{|c|}{ Up-regulated mRNAs } & \multicolumn{4}{|c|}{ Down-regulated mRNAs } \\
\hline Seqname & Gene symbol & Fold Change $^{a}$ & $\overline{\text { Chromosome }}$ & Seqname & Gene symbol & Fold Change $^{a}$ & Chromosome \\
\hline NM_013697 & Ttr & 18.8947104 & 18 & NM_010479 & Hspa1a & 9.6381486 & 17 \\
\hline NM_007976 & F5 & 9.4551777 & 1 & NM_001159671 & Rsph6a & 3.7486615 & 7 \\
\hline NM_001142706 & $\mathrm{Cfb}$ & 7.2709559 & 17 & NM_010478 & Hspa1b & 3.1997402 & 17 \\
\hline NM_024283 & 1500015010Rik & 6.9567191 & 1 & NM_026080 & Mrps24 & 2.7997696 & 11 \\
\hline NM_010030 & Defb2 & 6.2209284 & 8 & NM_001164259 & Fgfrl1 & 2.7403876 & 5 \\
\hline NM_020516 & Slc16a8 & 5.5709692 & 15 & NM_177294 & Rpap1 & 2.7259814 & 2 \\
\hline NM_009902 & Cldn3 & 4.8754256 & 5 & NM_001099328 & Zfp831 & 2.722746 & 2 \\
\hline NM_139221 & Defb11 & 4.863742 & 8 & NM_026159 & Retsat & 2.6378435 & 6 \\
\hline NM_001033233 & Tmprss11a & 4.7814956 & 5 & NM_013604 & Mtx1 & 2.5202344 & 3 \\
\hline NM_013823 & $\mathrm{Kl}$ & 4.5972095 & 5 & NM_001163667 & Tnnt3 & 2.4143907 & 7 \\
\hline
\end{tabular}

Notes: ${ }^{a}$ The SUDEP group compared with normal group

This study also revealed fifteen dysregulated long intergenic noncoding RNAs (lincRNAs) and their associated mRNAs, of which the most aberrantly expressed lincRNA was upregulated AK013439, and its corresponding mRNAs were Gnas (downregulated) and Zfp831 (downregulated). The results are presented in the supplementary material (see Additional file 1: Table 2).

\section{Quantitative real-time PCR validation}

Four IncRNAs (uc007urg.1, AK029922, ENSMUST00000152600, ENSMUST00000172531) were selected for further validation by qRT-PCR. The results showed that all these lncRNAs exhibited significant differences $(P$ value $<0.05)$ between the two groups, which was in accordance with the microarray results (Fig. 5).

\section{Discussion}

Over the past decades, an increasing number of studies have explored the potential molecular and electrophysiological mechanisms of SUDEP. Recently, increased awareness has been given to SUDEP-related genes, which have mostly been identified as neurocardiac genes (i.e., KCNA1, SCN1A, SCN8A, KCNQ1) [12, 27]. However, although some SUDEP cases have a genetic background, a large proportion of cases have not carried clear genetic mutations [28]. This suggests that SUDEP is a highly heterogenic disease in genetics and requires further study in different aspects.

However, much less is known about the changes and roles of mRNAs and noncoding RNAs (ncRNAs) in SUDEPs. In Scorza et al.'s study, they first reported that microRNAs might participate in SUDEP [29]. De Matteis et al. identified miR-301a-3p as an innovative potential biomarker in SUDEP [30]. Other ncRNAs, including lncRNAs, are still poorly understood in SUDEP. Inspired by recent findings on pathology that repeat seizures lead to intermediate structural changes between normal brainstem and SUDEP cases [8], we speculated that there is an epigenetic regulation process that alters the structure and function of the brainstem.

To further explore the potential role of lncRNAs and epigenetic regulation in SUDEP, we studied the susceptibility gaining process in a DBA/1 mouse SUDEP model. We found a total of 502 lncRNAs and 263 mRNAs dysregulated between the SUDEP model and normal DBA/1 control. On the mRNA expression profile, we first tried to establish a relation between differentially expressed mRNAs according to known hypotheses related to SUDEP. According to a previous SUDEP mechanism study on this model, fewer serotonin (5-HT) receptors characterized S-IRA-prone mice [23]. However, we did not find any changes in the mRNA of these receptors. This suggests that the difference in the 5-HT system was not made by priming and thus more likely to be intrinsic than epigenetic. Similarly, there was no evidence from our data that any specific ion channel was related to SUDEP. On the other hand, we found significant downregulation of the adenosine A3 receptor. Recently, an imbalance of adenosine receptors in the cortex has been proposed to increase the risk for SUDEP [31]. Adenosine itself is a strong anticonvulsant, while overaccumulation of adenosine could induce respiratory arrest and apnea, thus participating in SUDEP [32]. However, there have been no reports on the relationship of adenosine A3 receptors to epilepsy or SUDEP. In other studies, adenosine A3 receptor has broad functions, including classical functions as a $\mathrm{G}$ protein-coupled receptor, inhibiting calcium current and novel functions when binding with $\mathrm{N}$ ${ }^{6}$-methyladenosine (m6A) [33, 34]. Further mechanistic studies are needed to establish its linkage on SUDEP.

In coexpression analysis, both antisense lncRNAs and lincRNAs exhibited differential expression. Antisense lncRNAs serve critical functions in gene expression by 


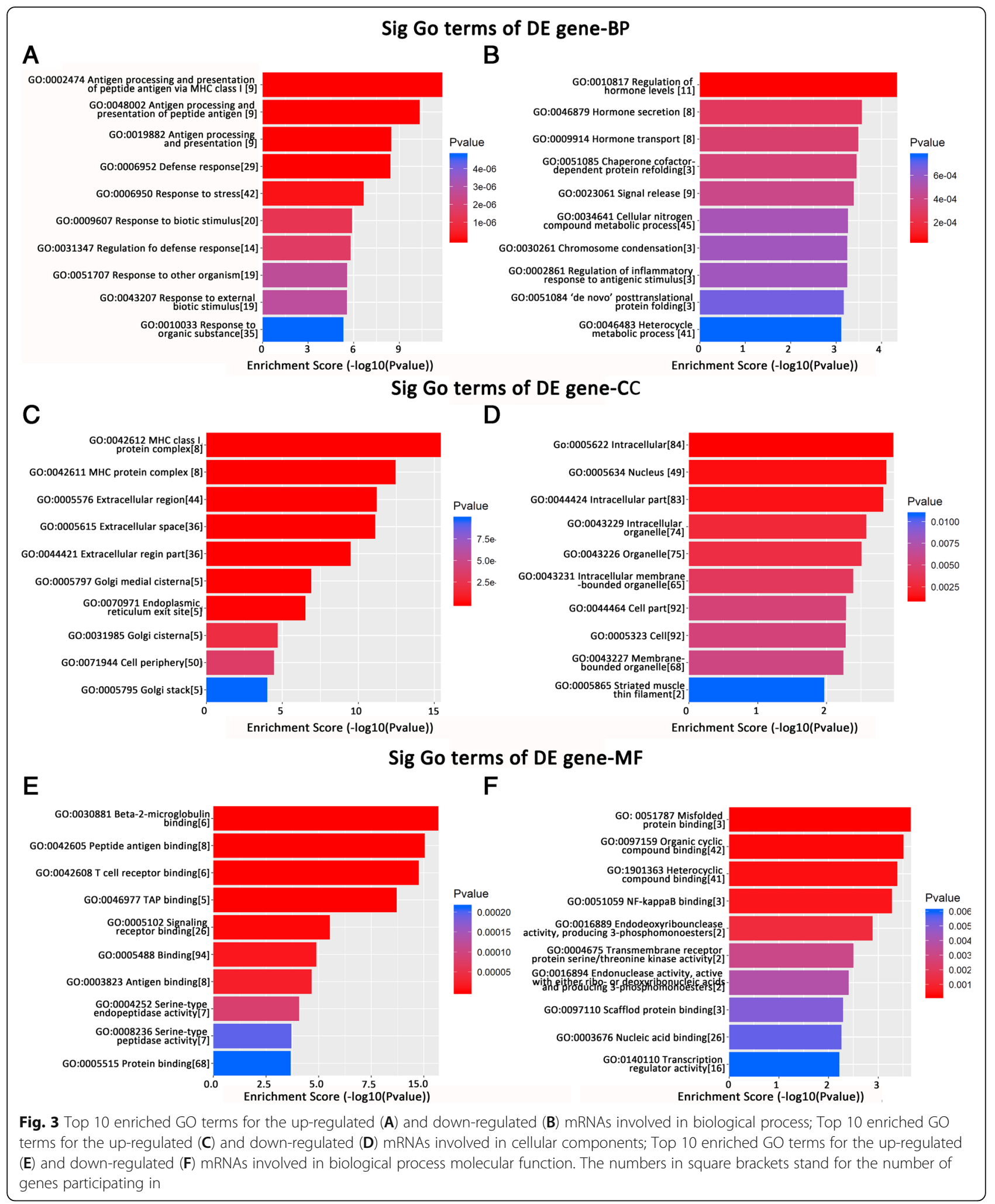

regulating transcriptional or posttranscriptional processes [35]. Three natural antisense lncRNAs (AK012034, AK032393, and uc007ovn.2) were found to be differentially expressed in the current study. The antisense mRNA of AK012034 was identified as Lmnb2, which presented a 1.78-fold downregulation in the SUDEP group. It has been reported that a mutation in $L M N B 2$ could lead to progressive myoclonus epilepsy 


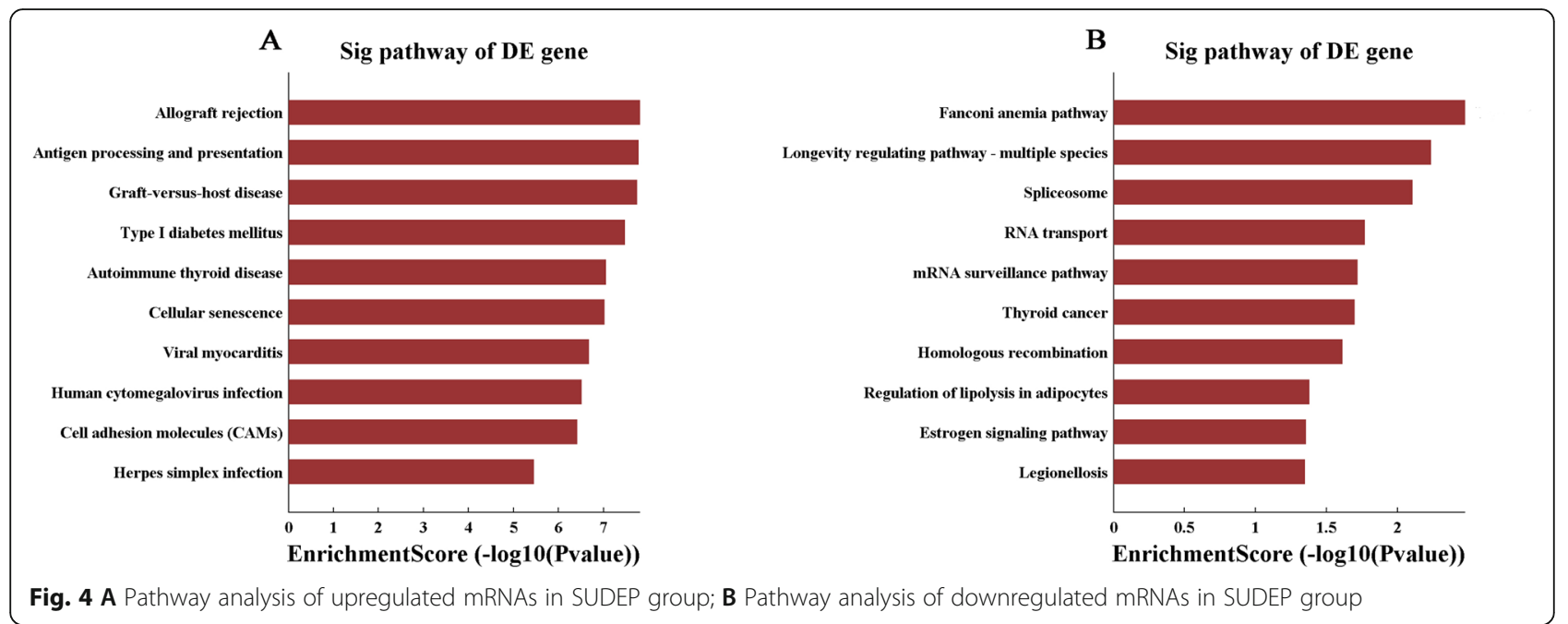

[36, 37]. This finding implied a potential epigenetic regulation of susceptibility to SUDEP through AK012034 and Lmnb2.

LincRNAs are another subgroup of lncRNAs that have been observed to have regulatory roles at the transcriptional and epigenetic levels [38]. However, the function of most lincRNAs remains unknown. Fifteen dysregulated lincRNAs and adjacent coding mRNAs were identified in this study. Among these differences, lncRNA NR_040757 and its nearby gene ITPR1 could be related to SUDEP, as the mutation of ITPR1 has been found in SUDEP cases [39]. However, the underlying mechanism requires further study.

GO and KEGG pathway analyses addressed the biological functions of differentially expressed lncRNAs and mRNAs. In GO terms of biological process and
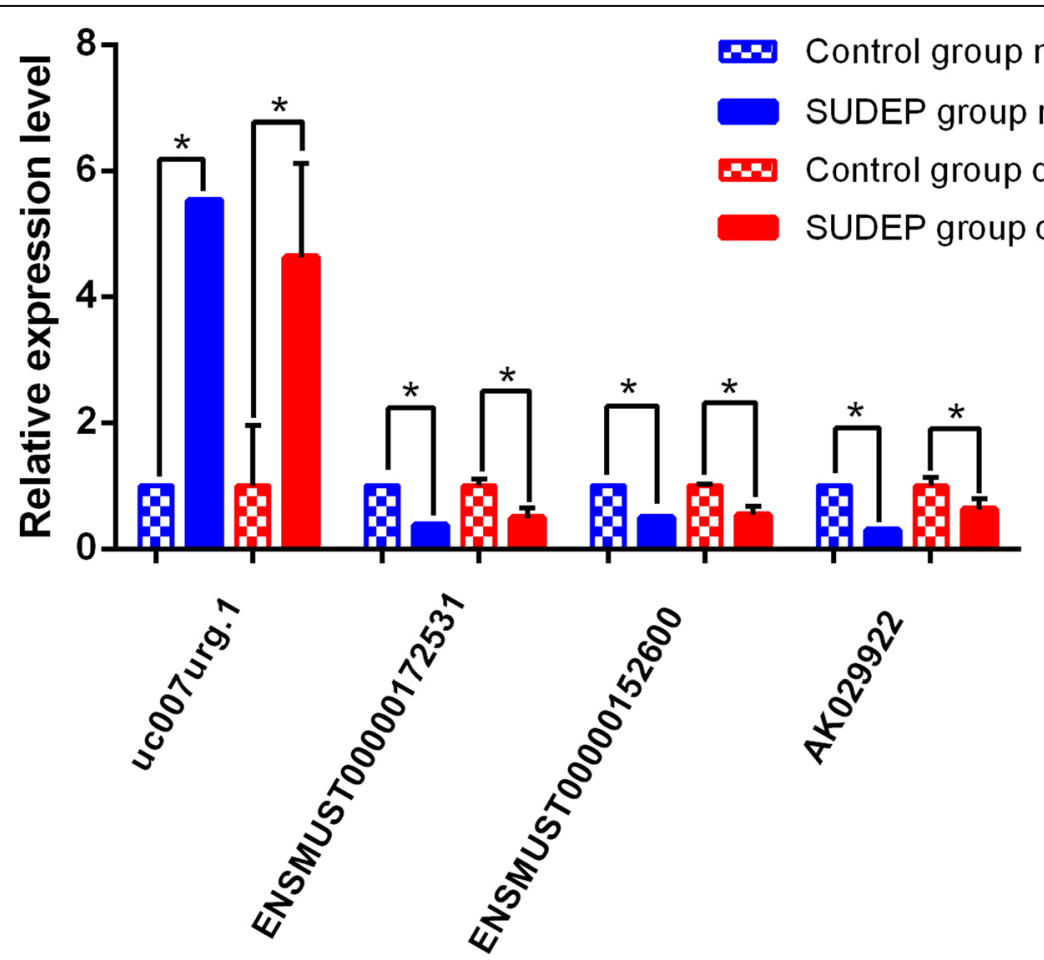

Fig. 5 The validation of differentially expressed IncRNAs. Note: The expression value of IncRNAs in control group was set at 1, while the corresponding expression level of which in SUDEP group was the fold change relative to control group. The qRT-PCR results were consistent with microarray data. Significant levels were indicated by * $(P<0.05)$ 
molecular function, we focused on chaperone cofactordependent protein refolding and misfolded protein binding, which was surprisingly found among the downregulated terms. The related mRNAs are Hspala and Hspa1b, and they encode HSP-70 (heat shock protein, $70 \mathrm{kDa}$ ). HSP-70 has been tested in a postmortem study of SUDEP cases and has been found to be upregulated in the hippocampus [40]. A recent study of proteomics and RNA sequencing also revealed similar patterns in SUDEP cases and mesial temporal lobe epilepsy (mTLE). HSP-70 was thus considered a marker of recent seizures prior to final death [41]. However, in our study, even though all sacrificed mice suffered from seizures $24 \mathrm{~h}$ prior to death, we found downregulation of Hspala and Hspalb with high downregulation of the regulatory fold change (Hspa1a 9.64 down, Hspa1b3.20 down). Considering its biological function, the unusual downregulation of HSP may represent suppression of the stress response [42] and misfolding of protein processing. These changes could further influence apoptosis $[43,44]$ and potentially change the composition and distribution of neurons in the brainstem, thus participating in SUDEP [8]. The mechanism of such downregulation remained unclear, although the coexpression analysis indicated that there is a lncRNA ENSMUST00000172531 adjacent to both HSPA1A and HSPA1B with a fold change of 2.61. It is likely that this lncRNA acts as an epigenetic regulating factor for the downregulation of these two genes. The KEGG analysis suggested several deregulated pathways, although they do not match the known mechanisms of S-IRA and SUDEP. Further study is still needed to confirm these findings.

Due to the difficulties in acquiring samples, studies on the expression profile and role of ncRNAs in SUDEPs are limited. In 2021, Leitner et al. reported an RNA sequencing study in SUDEP high-risk cases [45]. This study used hippocampal samples from mesial temporal lobe epilepsy surgery and found 55 dysregulated RNAs, including 37 mRNAs, 15 lncRNAs and 3 unknown RNAs. However, the published data only provided 20 mRNAs, and none of them overlapped with our dysregulated mRNAs or downstream targets of lncRNAs. Although the study used mTLE samples with a high risk of SUDEP, rather than real SUDEP, and the choice of hippocampal tissue is controversial, their study is the first and only attempt to systematically explore SUDEP from ncRNA prospects in humans. Christiansen et al. performed another epigenetic analysis by testing the methylation on the genome between sudden unexplained death cases and SUDEP. Their study found 6 different methylation regions near glutathione S-transferase (GST) superfamily genes, and the expression of GSTT1 was negatively related to the methylation of GST [46]. In our study, we identified that GSTT4 was downregulated (fold change $1.51, P=0.027$ ). By a similar mechanism to GSTT1, it may potentially influence the methylation of nearby genes and thus participate in SUDEP.

Our study has some limitations. First, we manually controlled the interval between the last seizure and sacrifice as $24 \mathrm{~h}$, leaving long-term changes unavailable. Second, the tissue lacked anatomical precision, as the whole brainstem was used in the extraction of RNAs. Third, the heterogeneity of lncRNAs between humans and mice makes some of the results inapplicable for human studies.

\section{Conclusions}

Abnormal expression of lncRNAs and dysregulated mRNAs was found in the DBA/1 SUDEP model compared to the normal control. Several RNAs, including Adora3, Lmnb2, Hspa1a, Hspa1b, Itrp1, Gstt4 and their related lncRNAs, were proposed as candidates related to SUDEP. The detailed mechanism of these dysregulated mRNAs and lncRNAs in SUDEP requires further study.

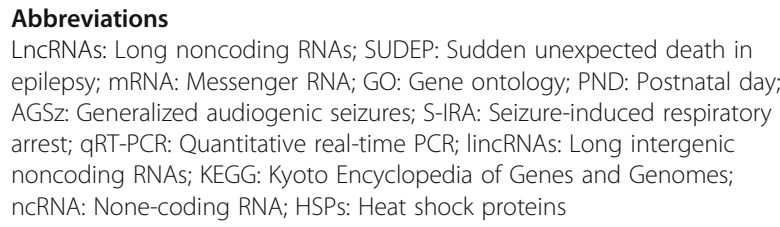

\section{Supplementary Information}

The online version contains supplementary material available at https://doi. org/10.1186/s12864-021-07921-7.

Additional file 1: Table 1. Differentially expressed antisense IncRNAs and nearby coding gene. ${ }^{*}$ The SUDEP group compared with normal group. Table 2. Differentially expressed lincRNAs and adjacent mRNAs. lincRNAs, long intergenic noncoding RNAs.*The SUDEP group compared with normal group.

Additional file 2. Details of the corresponding probe and region of each lincRNA

\section{Acknowledgements}

We thank KangChen Bio-tech, Shanghai, China for providing the microarray service.

\section{Authors' contributions}

DC designed the experiment and wrote the manuscript. LNZ and XL performed the experiments. DZ provided ideas for the manuscript and wrote the manuscript. LL gave the general idea, supervised the project, participated in writing and provided funding for the entire project. All authors read and approved the final manuscript.

\section{Funding}

This work was supported by the Science \& Technology Department of Sichuan Province [grant number 2018SZ0166]. The funder had no role in study design, data analysis, decision to publish, and preparation of the manuscript.

\section{Availability of data and materials}

The original datasets analyzed in the current study are available in the Gene Expression Omnibus (GEO) repository (GSE160576). (https://www.ncbi.nlm. nih.gov/geo/query/acc.cgi?acc=GSE160576).

The reference datasets include ( $X$ refers to any number or letter.): 
NCBI RefSeq database (NR_XXXXXX or NM_XXXXXX) (https://www.ncbi.nlm. nih.gov/refseg/)

Ensembl (ENSMUSTXXXXXXXXXXX) (http://www.ensembl.org/index.html); mRNA or EST records in Genbank without coding evidence (AKXXXXXX) (https://www.ncbi.nlm.nih.gov/genbank/); UCSC known genes (uCXXXXXX.X) (http://genome.ucsc.edu/cgi-bin/hgTables/

"ultra-conserved region" among human, mouse and rat (uc.XXX) (http://users. soe.ucsc.edu/ jill/ultra.html);

lincRNAs identified by John Rinn's group (Guttman et al. [47], Khalil et al. [48]) are coded as 'humanlincRNAXXXX' or 'mouselincRNAXXXX' in this research according to species. The detail of the corresponding probe sequence and region for each lincRNA is in supplementary material (see Additional file 2).

\section{Declarations}

\section{Ethics approval and consent to participate}

The study was approved by the Ethics Committee of Sichuan University.

\section{Consent for publication}

Not applicable.

\section{Competing interests}

The authors declare that they have no competing interests.

\section{Received: 23 November 2020 Accepted: 3 August 2021} Published online: 17 August 2021

\section{References}

1. Ponting $C P$, Oliver PL, Reik W. Evolution and functions of long noncoding RNAs. Cell. 2009;136(4):629-41. https://doi.org/10.1016/j.cell.2009.02.006.

2. Clark MB, Mattick JS. Long noncoding RNAs in cell biology. Semin Cell Dev Biol. 2011;22(4):366-76. https://doi.org/10.1016/j.semcdb.2011.01.001.

3. Ng SY, Lin L, Soh BS, Stanton LW. Long noncoding RNAs in development and disease of the central nervous system. Trends Genet. 2013;29(8):461-8. https://doi.org/10.1016/j.tig.2013.03.002.

4. Lee DY, Moon J, Lee ST, et al. Dysregulation of long non-coding RNAs in mouse models of localization-related epilepsy. Biochem Biophys Res Commun. 2015;462(4):433-40. https://doi.org/10.1016/j.bbrc.2015.04.149.

5. Xiao W, Cao Y, Long H, et al. Genome-Wide DNA Methylation Patterns Analysis of Noncoding RNAs in Temporal Lobe Epilepsy Patients. Mol Neurobiol. 2018;55(1):793-803. https://doi.org/10.1007/s12035-016-0353-X.

6. Hsiao J, Yuan TY, Tsai MS, et al. Upregulation of Haploinsufficient Gene Expression in the Brain by Targeting a Long Non-coding RNA Improves Seizure Phenotype in a Model of Dravet Syndrome. EBioMedicine. 2016;9: 257-77. https://doi.org/10.1016/j.ebiom.2016.05.011.

7. Hughes JR. A review of sudden unexpected death in epilepsy: prediction of patients at risk. Epilepsy Behav. 2009;14(2):280-7. https://doi.org/10.1016/j. yebeh.2008.12.004

8. Patodia S, Somani A, O'Hare M, et al. The ventrolateral medulla and medullary raphe in sudden unexpected death in epilepsy. Brain. 2018;141(6): 1719-33. https://doi.org/10.1093/brain/awy078.

9. Mueller SG, Bateman LM, Laxer KD. Evidence for brainstem network disruption in temporal lobe epilepsy and sudden unexplained death in epilepsy. Neuroimage Clin. 2014;5:208-16. https://doi.org/10.1016/j.nicl.2014. 06.010 Published 2014 Jul 9.

10. Mueller SG, Nei M, Bateman LM, et al. Brainstem network disruption: A pathway to sudden unexplained death in epilepsy? Hum Brain Mapp. 2018. 39(12):4820-30. https://doi.org/10.1002/hbm.24325.

11. Devinsky O, Hesdorffer DC, Thurman DJ, Lhatoo S, Richerson G. Sudden unexpected death in epilepsy: epidemiology, mechanisms, and prevention. Lancet Neurol. 2016;15(10):1075-88. https://doi.org/10.1016/S1474-4422(16)3 0158-2.

12. Glasscock E. Genomic biomarkers of SUDEP in brain and heart. Epilepsy Behav. 2014;38:172-9. https://doi.org/10.1016/j.yebeh.2013.09.019.

13. Lhatoo SD, Faulkner HJ, Dembny K, Trippick K, Johnson C, Bird JM. An electroclinical case-control study of sudden unexpected death in epilepsy. Ann Neurol. 2010;68(6):787-96. https://doi.org/10.1002/ana.2210.

14. Ryvlin $\mathrm{P}$, Nashef $\mathrm{L}$, Lhatoo SD, et al. Incidence and mechanisms of cardiorespiratory arrests in epilepsy monitoring units (MORTEMUS): a retrospective study. Lancet Neurol. 2013;12(10):966-77. https://doi.org/10.1 016/S1474-4422(13)70214-X.

15. Harden C, Tomson T, Gloss D, et al. Practice guideline summary: Sudden unexpected death in epilepsy incidence rates and risk factors: Report of the Guideline Development, Dissemination, and Implementation Subcommittee of the American Academy of Neurology and the American Epilepsy Society. Neurology. 2017;88(17):1674-80. https://doi.org/10.1212/WNL.

0000000000003685 [published correction appears in Neurology. 2019 Nov 26;93(22):982] [published correction appears in Neurology. 2020 Mar 3;94(9): 414].

16. Chen D, Si Y, He J, et al. Terminal seizure frequency and its relation to SUDEP. Epilepsy Behav. 2017;70(Pt A):173-6. https://doi.org/10.1016/j. yebeh.2017.02.002

17. Feng $\mathrm{HJ}$, Faingold $\mathrm{CL}$. Abnormalities of serotonergic neurotransmission in animal models of SUDEP. Epilepsy Behav. 2017;71(Pt B):174-80. https://doi. org/10.1016/j.yebeh.2015.06.008.

18. Langan $Y$, Nashef L, Sander JW. Sudden unexpected death in epilepsy: a series of witnessed deaths. J Neurol Neurosurg Psychiatry. 2000;68(2):211-3. https://doi.org/10.1136/jnnp.68.2.211.

19. So EL, Sam MC, Lagerlund TL. Postictal central apnea as a cause of SUDEP: evidence from near-SUDEP incident. Epilepsia. 2000;41(11):1494-7. https:// doi.org/10.1111/j.1528-1157.2000.tb00128.x.

20. Bateman LM, Li CS, Seyal M. Ictal hypoxemia in localization-related epilepsy: analysis of incidence, severity and risk factors. Brain. 2008;131(Pt 12):323945. https://doi.org/10.1093/brain/awn277.

21. Blum AS. Respiratory physiology of seizures. J Clin Neurophysiol. 2009;26(5): 309-15. https://doi.org/10.1097/WNP.0b013e3181b7f14d.

22. Pezzella M, Striano P, Ciampa C, Errichiello L, Penza P, Striano S. Severe pulmonary congestion in a near miss at the first seizure: further evidence for respiratory dysfunction in sudden unexpected death in epilepsy. Epilepsy Behav. 2009;14(4):701-2. https://doi.org/10.1016/j.yebeh.2009.02. 012.

23. Zhang H, Zhao H, Yang X, Xue Q, Cotten JF, Feng HJ. 5-Hydroxytryptophan, a precursor for serotonin synthesis, reduces seizure-induced respiratory arrest. Epilepsia. 2016;57(8):1228-35. https://doi.org/10.1111/epi.13430.

24. Kommajosyula SP, Randall ME, Brozoski TJ, Odintsov BM, Faingold CL. Specific subcortical structures are activated during seizure-induced death in a model of sudden unexpected death in epilepsy (SUDEP): A manganeseenhanced magnetic resonance imaging study. Epilepsy Res. 2017;135:87-94. https://doi.org/10.1016/j.eplepsyres.2017.05.011.

25. Zhang $\mathrm{H}$, Zhao $\mathrm{H}$, Zeng $\mathrm{C}$, et al. Optogenetic activation of 5-HT neurons in the dorsal raphe suppresses seizure-induced respiratory arrest and produces anticonvulsant effect in the DBA/1 mouse SUDEP model. Neurobiol Dis. 2018;110:47-58. https://doi.org/10.1016/j.nbd.2017.11.003.

26. Faingold $\mathrm{CL}$, Randall M, Tupal S. DBA/1 mice exhibit chronic susceptibility to audiogenic seizures followed by sudden death associated with respiratory arrest. Epilepsy Behav. 2010;17(4):436-40. https://doi.org/10.1016/j.yebeh.201 0.02.007.

27. Coll M, Oliva A, Grassi S, Brugada R, Campuzano O. Update on the Genetic Basis of Sudden Unexpected Death in Epilepsy. Int J Mol Sci. 2019;20(8): 1979. https://doi.org/10.3390/ijms20081979 Published 2019 Apr 23.

28. Ge Y, Ding D, Zhu G, et al. Genetic variants in incident SUDEP cases from a community-based prospective cohort with epilepsy. J Neurol Neurosurg Psychiatry. 2020;91(2):126-31. https://doi.org/10.1136/jnnp-2019-321983.

29. Scorza FA, Cendes F, Cavalheiro EA, Lopes-Cendes I. Sudden unexpected death in epilepsy: small RNAs raise expectations. Epilepsy Behav. 2013;29(3): 591-3. https://doi.org/10.1016/j.yebeh.2013.08.009.

30. De Matteis M, Cecchetto G, Munari G, et al. Circulating miRNAs expression profiling in drug-resistant epilepsy: Up-regulation of miR-301a-3p in a case of sudden unexpected death. Leg Med (Tokyo). 2018;31:7-9. https://doi. org/10.1016/j.legalmed.2017.12.003

31. Patodia S, Paradiso B, Garcia M, et al. Adenosine kinase and adenosine receptors $A 1 R$ and $A 2 A R$ in temporal lobe epilepsy and hippocampal sclerosis and association with risk factors for SUDEP. Epilepsia. 2020;61(4): 787-97. https://doi.org/10.1111/epi.16487.

32. Weltha $L$, Reemmer J, Boison D. The role of adenosine in epilepsy. Brain Res Bull. 2019;151:46-54. https://doi.org/10.1016/j.brainresbull.2018.11.008.

33. Coppi $E_{1}$ Cherchi F, Fusco I, et al. Adenosine A3 receptor activation inhibits pronociceptive $\mathrm{N}$-type Ca2 + currents and cell excitability in dorsal root ganglion neurons. Pain. 2019;160(5):1103-18. https://doi.org/10.1097/j.pain. 0000000000001488 
34. Ogawa A, Nagiri C, Shihoya W, et al. N6-methyladenosine ( $\mathrm{m} 6 \mathrm{~A})$ is an endogenous A3 adenosine receptor ligand. Mol Cell. 2021;81(4):659-74.e7. https://doi.org/10.1016/j.molcel.2020.12.038.

35. Vadaie N, Morris KV. Long antisense non-coding RNAs and the epigenetic regulation of gene expression. Biomol Concepts. 2013;4(4):411-5. https:// doi.org/10.1515/bmc-2013-0014.

36. Damiano JA, Afawi Z, Bahlo M, et al. Mutation of the nuclear lamin gene LMNB2 in progressive myoclonus epilepsy with early ataxia. Hum Mol Genet. 2015;24(16):4483-90. https://doi.org/10.1093/hmg/ddv171.

37. Soleimanipour F, Razmara E, Rahbarizadeh F, et al. A novel missense variant in the LMNB2 gene causes progressive myoclonus epilepsy [published online ahead of print, 2021 Mar 30]. Acta Neurol Belg. 2021. https://doi. org/10.1007/s13760-021-01650-0.

38. Deniz E, Erman B. Long noncoding RNA (lincRNA), a new paradigm in gene expression control. Funct Integr Genomics. 2017;17(2-3):135-43. https://doi. org/10.1007/s10142-016-0524-X.

39. Friedman D, Kannan K, Faustin A, et al. Cardiac arrhythmia and neuroexcitability gene variants in resected brain tissue from patients with sudden unexpected death in epilepsy (SUDEP). NPJ Genom Med. 2018;3:9. https://doi.org/10.1038/s41525-018-0048-5 Published 2018 Mar 27.

40. Thom M, Seetah S, Sisodiya S, Koepp M, Scaravilli F. Sudden and unexpected death in epilepsy (SUDEP): evidence of acute neuronal injury using HSP-70 and c-Jun immunohistochemistry. Neuropathol Appl Neurobiol. 2003;29(2):132-43. [https://doi.org/10.1046/j.1365-2990.2003.004 52.x].

41. Leitner DF, Mills JD, Pires G, et al. Proteomics and Transcriptomics of the Hippocampus and Cortex in SUDEP and High-Risk SUDEP Patients. Neurology. 2021;96(21):e2639-52. https://doi.org/10.1212/WNL. 0000000000011999

42. Sõti C, Nagy E, Giricz Z, Vígh L, Csermely P, Ferdinandy P. Heat shock proteins as emerging therapeutic targets. Br J Pharmacol. 2005;146(6):76980. https://doi.org/10.1038/sj.bjp.0706396.

43. Sottile ML, Nadin SB. Heat shock proteins and DNA repair mechanisms: an updated overview. Cell Stress Chaperones. 2018;23(3):303-15. https://doi. org/10.1007/s12192-017-0843-4.

44. Macario AJ, Conway de Macario E. Molecular chaperones: multiple functions, pathologies, and potential applications. Front Biosci. 2007;12: 2588-600. https://doi.org/10.2741/2257 Published 1 Jan 2007.

45. Leitner DF, Mills JD, Pires G, et al. Proteomics and Transcriptomics of the Hippocampus and Cortex in SUDEP and High-Risk SUDEP Patients. Neurology. 2021;96(21):e2639-e2652. https://doi.org/10.1212/WNL. 0000000000011999

46. Christiansen SN, Jacobsen SB, Andersen JD, et al. Differential Methylation in the GSTT1 Regulatory Region in Sudden Unexplained Death and Sudden Unexpected Death in Epilepsy. Int J Mol Sci. 2021;22(6):2790. https://doi. org/10.3390/ijms22062790 Published 10 Mar 2021.

47. Guttman M, Amit I, Garber M, et al. Chromatin signature reveals over a thousand highly conserved large non-coding RNAs in mammals. Nature. 2009;458(7235):223-7. https://doi.org/10.1038/nature07672.

48. Khalil AM, Guttman M, Huarte M, et al. Many human large intergenic noncoding RNAs associate with chromatin-modifying complexes and affect gene expression. Proc Natl Acad Sci U S A. 2009;106(28):11667-72. https:// doi.org/10.1073/pnas.0904715106.

\section{Publisher's Note}

Springer Nature remains neutral with regard to jurisdictional claims in published maps and institutional affiliations.

Ready to submit your research? Choose BMC and benefit from:

- fast, convenient online submission

- thorough peer review by experienced researchers in your field

- rapid publication on acceptance

- support for research data, including large and complex data types

- gold Open Access which fosters wider collaboration and increased citations

- maximum visibility for your research: over $100 \mathrm{M}$ website views per year

At BMC, research is always in progress.

Learn more biomedcentral.com/submissions 